\title{
Clinical utility of gadobenate dimeglumine in contrast-enhanced MRI of the breast: a review
}

This article was published in the following Dove Press journal:

Research and Reports in Nuclear Medicine

25 November 2015

Number of times this article has been viewed

\author{
Maria Laura Luciani' \\ Marianna Telesca' \\ Miles A Kirchin ${ }^{2}$ \\ Federica Pediconi' \\ 'Department of Radiological Sciences, \\ Oncology and Anatomic Pathology, \\ Sapienza University of Rome, \\ Rome, Italy; ${ }^{2}$ Global Medical \& \\ Regulatory Affairs, Bracco Imaging \\ SpA, Milan, Italy
}

\begin{abstract}
Breast magnetic resonance imaging (MRI) is considered the technique with the highest sensitivity for breast cancer detection. Gadobenate dimeglumine is a gadolinium-based contrast agent (GBCA) that is specifically approved in Europe for breast MRI and which has the highest $\mathrm{r} 1$ relaxivity among all GBCAs for this indication. In order to improve the diagnostic performance of breast MRI, several intra-individual crossover studies have evaluated gadobenate dimeglumine as a possible GBCA for this application. This review focuses on the role and advantages of gadobenate dimeglumine as a contrast agent for breast MRI by describing the unique properties of this agent and by summarizing published studies.

Keywords: magnetic resonance, breast imaging, oncology, breast, tumor, women's imaging
\end{abstract}

\section{Introduction}

Breast cancer is the most common cancer among women, accounting for over one fifth of the estimated annual 4.7 million cancer diagnoses in females. It is the main cause of death from cancer in women., ${ }^{1,2}$ Conventional X-ray mammography and ultrasound are the most widely used techniques for breast imaging but have comparatively low sensitivity and specificity for dense breast evaluation or in patients with breast implants, post-surgical scars or deformity. ${ }^{2-7}$ Of the techniques available, magnetic resonance imaging (MRI) has consistently demonstrated superior sensitivity to conventional imaging techniques for breast cancer detection. ${ }^{8,9}$ In this regard, a recent meta-analysis of 44 studies revealed an overall sensitivity of 0.90 for MRI in the evaluation of breast cancer. ${ }^{10}$ Although specificity was somewhat lower at 0.72 , this reflected the inclusion of several studies published during the early days of breast MRI when specificity was still an issue.

In recent years the role of breast MRI has increased dramatically. Among the many applications for breast MRI are detection of primary and recurrent breast cancer, screening for high-risk women, evaluation of the response to neoadjuvant chemotherapy, preoperative staging, and assessment of breast implants. ${ }^{11-17}$

MRI is advantageous for breast imaging because of high soft-tissue contrast and the possibility for multiplanar sectioning which permits full three-dimensional representation of one or both breasts. With recent technological improvements in imaging technique, as well as standardization of image acquisition and interpretation, breast MRI enhanced with gadolinium-based contrast agents (GBCAs) is now widely accepted as an integral part of clinical practice at most centers. ${ }^{18}$
Correspondence: Maria Laura Luciani Department of Radiological Sciences, Oncology and Anatomic Pathology, Sapienza University of Rome,Viale Regina Elena 324, 0016I Rome, Italy

Email marialaura.luciani@uniromal.it 


\section{GBCAs}

Gadolinium is a rare earth metal which belongs to the lanthanide family of elements; it is a highly paramagnetic element having seven unpaired electrons which make it highly suitable for MRI. ${ }^{19}$ As free gadolinium is highly toxic, all GBCAs consist of gadolinium bound to an organic chelating molecule. The GBCAs available for breast MRI have an extracellular distribution with a half-life of elimination of approximately 1.5-2.0 hours in patients with normal renal function. ${ }^{19,20}$ The approved contrast agent dose is $0.1 \mathrm{mmol} / \mathrm{kg}$ of body weight which is usually injected at a rate of $2-3 \mathrm{~mL} / \mathrm{s}^{19,20}$

GBCAs shorten the $\mathrm{T} 1, \mathrm{~T} 2$, and $\mathrm{T} 2 *$ relaxation time constants of adjacent water protons in tissues producing signal enhancement on T1-weighted MR images and signal loss on T2- and T2*-weighted MR images. ${ }^{19}$ The extent to which a GBCA shortens the relaxation times depends on the relaxivity of the agent. GBCAs have different structures and physicochemical properties but most have similar $\mathrm{r} 1$ relaxivity values of 3.9-4.6 L/mmols ${ }^{-1}$ at $1.5 \mathrm{~T}^{21}$ Of the GBCAs available for breast imaging gadobenate dimeglumine (MultiHance ${ }^{\circledR}$; Bracco imaging, Milan, Italy) has a markedly higher $\mathrm{r} 1$ relaxivity in vivo of $6.2 \mathrm{~L} / \mathrm{mmols}^{-1}$ at $1.5 \mathrm{~T}$.

Several studies have demonstrated an association between the use of contrast agents with higher $\mathrm{r} 1$ relaxivity and stronger contrast enhancement in comparison to contrast agents with lower $r 1$ relaxivity. For most clinical applications, the stronger enhancement has been shown to be associated with a clinically relevant gain in diagnostic performance..$^{22-26}$

\section{GBCAs and breast MRI}

The first GBCA utilized for breast MRI was gadopentetate dimeglumine. ${ }^{27}$ Since then several other GBCAs have been evaluated. Renz et al evaluated 45 patients with gadoteric acid and gadobutrol and while some differences were noted in terms of the dynamic and morphologic characterization of breast lesions, both GBCAs were considered reliable for breast MRI. ${ }^{28}$ More recently, Fallenberg et al performed an intra-individual comparison of the same two GBCAs and observed a stronger relative signal intensity enhancement with gadobutrol. However, no significant differences were noted in terms of the characterization of breast lesions. ${ }^{29}$

\section{Gadobenate dimeglumine (MultiHance) in breast MRI}

Gadobenate dimeglumine differs from other GBCAs approved for breast MRI in that it possesses a benzyloxymethyl substituent on the chelate structure. This substituent causes the gadobenate molecule to interact weakly, transiently, and non-covalently with human serum albumin, a principal consequence of which is a slowing of the tumbling rate of the gadolinium complex and thus greater relaxivity (and hence shorter $\mathrm{T} 1, \mathrm{~T} 2$, and $\mathrm{T} 2 *$ relaxation times) than GBCAs that do not interact with serum albumin. ${ }^{30}$

Since neoangiogenesis in breast cancer is associated with increased vascularity and permeability, breast malignancies typically show higher contrast enhancement than normal breast parenchyma following GBCA administration. The greater $\mathrm{r} 1$ relaxivity of gadobenate dimeglumine deriving from interaction with serum albumin is therefore potentially highly beneficial for the improved detection of malignant breast lesions with abnormal vascular permeability in which the concentration of serum albumin is likely to be increased.

Numerous advantages to the use of gadobenate dimeglumine for breast MRI have been described in the literature and a great deal of data are available on the diagnostic performance achievable with this GBCA (Table 1). ${ }^{22-26}$

An early study to establish the optimal dose of gadobenate dimeglumine to use for breast MRI established that $0.1 \mathrm{mmol} / \mathrm{kg}$ of bodyweight is superior to doses of 0.05 and $0.2 \mathrm{mmol} / \mathrm{kg}$ of bodyweight in terms of lesion detection in women with known or suspected breast cancer. ${ }^{22}$ Furthermore, $0.1 \mathrm{mmol} / \mathrm{kg}$ gadobenate dimeglumine was also shown to be significantly superior to an equivalent dose of gadopentetate dimeglumine, not only in terms of malignant lesion detection but also for quantitative contrast enhancement and sensitivity for lesion characterization, meaning that detected lesions were easier to see and classify. Importantly, fewer false positive determinations were made with $0.1 \mathrm{mmol} / \mathrm{kg}$ gadobenate dimeglumine than with $0.1 \mathrm{mmol} / \mathrm{kg}$ gadopentetate dimeglumine. Unfortunately, a limitation of dose-finding studies of this type is that they necessarily have an inter-individual design in which patients receive just one contrast-enhanced examination and results are compared between groups. Such studies are subject to wide between-group variability in terms of patient population, breast density, size and type of lesions etc, making it difficult to accurately adjudicate the contrast efficacy of one agent against another.

A more accurate comparison of contrast agent efficacy is achieved with intra-individual crossover studies in which patients receive both contrast agents in two otherwise identical MRI examinations separated by just a few days. A first study of this type with gadobenate dimeglumine in breast MRI was performed by Pediconi et $\mathrm{al}^{23}$ in 2005 in 26 women with suspected breast cancer based on findings from conventional imaging. Significantly superior detection of breast lesions and more accurate identification of malignant lesions were achieved with $0.1 \mathrm{mmol} / \mathrm{kg}$ gadobenate dimeglumine than with $0.1 \mathrm{mmol} / \mathrm{kg}$ gadopentetate dimeglumine. 
Table I Breast MRI: cross-over studies between gadobenate dimeglumine and other GBCAs

\begin{tabular}{|c|c|c|c|c|}
\hline Reference & Indication & Design of study & Comparators/number of patients & Results \\
\hline Knopp et $\mathrm{al}^{22}$ & Breast cancer & Inter-individual & $\begin{array}{l}\text { Gadobenate dimeglumine } \\
0.05-0.1-0.2 \mathrm{mmol} / \mathrm{kg} \\
\text { Gadopentetate dimeglumine } \\
0.1 \mathrm{mmol} / \mathrm{kg} \\
189 \text { patients }\end{array}$ & $\begin{array}{l}\text { Superiority of gadobenate dimeglumine not only } \\
\text { in terms of malignant lesion detection but also } \\
\text { in terms of sensitivity for lesion characterization }\end{array}$ \\
\hline Pediconi et $\mathrm{al}^{23}$ & Breast cancer & Intra-individual & $\begin{array}{l}\text { Gadobenate dimeglumine } 0.1 \mathrm{mmol} / \mathrm{kg} \\
\text { Gadopentetate dimeglumine } 0.1 \mathrm{mmol} / \mathrm{kg} \\
26 \text { patients }\end{array}$ & $\begin{array}{l}\text { Gadobenate dimeglumine significantly superior } \\
\text { to gadopentetate dimeglumine - greater } \\
\text { sensitivity and accuracy in the detection of } \\
\text { breast cancer nodules }\end{array}$ \\
\hline Pediconi et $\mathrm{al}^{24}$ & Breast cancer & Intra-individual & $\begin{array}{l}\text { Gadobenate dimeglumine } 0.1 \mathrm{mmol} / \mathrm{kg} \\
\text { Gadopentetate dimeglumine } 0.1 \mathrm{mmol} / \mathrm{kg} \\
\text { double-blind, randomized, crossover, } \\
47 \text { patients }\end{array}$ & $\begin{array}{l}\text { Gadobenate dimeglumine significantly superior } \\
\text { to gadopentetate dimeglumine - greater accuracy } \\
\text { in the detection and characterization of breast } \\
\text { cancer nodules }\end{array}$ \\
\hline Martincich et $\mathrm{al}^{25}$ & Breast cancer & Intra-individual & $\begin{array}{l}\text { Gadobenate dimeglumine } 0.1 \mathrm{mmol} / \mathrm{kg} \\
\text { Gadopentetate dimeglumine } 0.1 \mathrm{mmol} / \mathrm{kg} \\
\text { double-blind, randomized, crossover, } \\
157 \text { patients }\end{array}$ & $\begin{array}{l}\text { MRI with gadobenate dimeglumine had } \\
\text { significantly better diagnostic performance } \\
\text { (greater sensitivity, specificity, and predictive } \\
\text { values) than MRI with gadopentetate dimeglumine }\end{array}$ \\
\hline Gilbert et $\mathrm{al}^{26}$ & Breast cancer & Intra-individual & $\begin{array}{l}\text { Gadobenate dimeglumine } 0.1 \mathrm{mmol} / \mathrm{kg} \\
\text { Gadopentetate dimeglumine } 0.1 \mathrm{mmol} / \mathrm{kg} \\
\text { double-blind, randomized, crossover, } \\
153 \text { patients }\end{array}$ & $\begin{array}{l}\text { Significantly better diagnostic performance on } \\
\text { MRI with gadobenate dimeglumine than on MRI } \\
\text { with gadopentetate dimeglumine and significantly } \\
\text { better breast cancer detection compared with } \\
\text { conventional imaging techniques }\end{array}$ \\
\hline Pediconi et $\mathrm{al}^{33}$ & Breast cancer & Intra-individual & $\begin{array}{l}\text { Gadobenate dimeglumine } \\
(0.5 \mathrm{M}) 0.1 \mathrm{mmol} / \mathrm{kg} \\
\text { Gadobutrol (I M) } 0.1 \mathrm{mmol} / \mathrm{kg}\end{array}$ & $\begin{array}{l}\text { Non-inferiority of gadobutrol in comparison } \\
\text { with gadobenate dimeglumine for breast lesion } \\
\text { detection }\end{array}$ \\
\hline
\end{tabular}

Abbreviations: MRI, magnetic resonance imaging; GBCAs, gadolinium-based contrast agents.

Specifically, MRI with gadobenate dimeglumine depicted significantly $(P=0.003)$ more lesions ( 45 of 46$)$ than did MRI with gadopentetate dimeglumine (36 of 46), and detected lesions were significantly $(P<0.001)$ more conspicuous with gadobenate dimeglumine. Likewise, confidence for lesion characterization was significantly $(P=0.031)$ greater with gadobenate dimeglumine. Comparison of the contrast agents for their ability to help identify malignant lesions revealed significant $(P=0.02)$ superiority for gadobenate dimeglumine: sensitivity, specificity, positive predictive value, negative predictive value, and overall accuracy for malignant lesion identification were, respectively, $94.7 \%, 100 \%, 100 \%, 80.0 \%$, and $95.6 \%$ with gadobenate dimeglumine and $76.3 \%, 100 \%$, $100 \%, 47.1 \%$, and $80.4 \%$ with gadopentetate dimeglumine. Quantitative evaluation of signal intensity-time curves revealed significantly $(P<0.001)$ greater lesion enhancement with gadobenate dimeglumine.

A similar study in 2008 in a larger number of patients revealed similar findings. ${ }^{24}$ In that study 47 women with 78 histologically proven breast cancers underwent breast MRI examinations with $0.1 \mathrm{mmol} / \mathrm{kg}$ gadobenate dimeglumine and $0.1 \mathrm{mmol} / \mathrm{kg}$ gadopentetate dimeglumine. Again, gadobenate dimeglumine provided significantly better performance for the detection of breast lesions ( $75 / 78$ lesions detected in total with gadobenate dimeglumine compared with 62/78 lesions detected with gadopentetate dimeglumine; 49/50 malignant lesions detected with gadobenate dimeglumine compared with 38/50 malignant lesions detected with gadopentetate dimeglumine) and provided significantly better diagnostic performance (sensitivity $98.0 \%$ vs $76.0 \%$; specificity $71.4 \%$ vs $57.1 \%$; accuracy $88.5 \%$ vs $69.2 \%$; positive predictive value $86.0 \%$ vs $76.0 \%$; and negative predictive value $95.2 \%$ vs $57.1 \%$ ).

Based on these single-center studies a much larger clinical trial was performed in order to obtain marketing authorization for gadobenate dimeglumine for breast MRI in Europe. This trial (the DETECT trial, a multicenter, double-blind, randomized, intra-individual, crossover comparison between gadobenate dimeglumine and gadopentetate dimeglumine for breast MRI ${ }^{25}$ prospectively randomized 162 patients at 17 sites in Europe and People's Republic of China between 2007 and 2009 to undergo two breast MRI exams, one with gadobenate dimeglumine and the other with gadopentetate dimeglumine, each at a dose of $0.1 \mathrm{mmol} / \mathrm{kg}$ of body weight.

Overall 157/162 women received gadobenate dimeglumine and 155/162 women received gadopentetate dimeglumine. The three readers of the study reported significantly superior cancer detection with gadobenate dimeglumine. This superiority was noted for all malignant lesions including non-invasive carcinomas (gadobenate dimeglumine detected 17 non-invasive cancers while gadopentetate 
dimeglumine identified only eleven of the 17 cancers detected by gadobenate dimeglumine). Readers preferred gadobenate dimeglumine over gadopentetate dimeglumine for determinations of lesion conspicuity and lesion border delineation. Moreover the rate of cancer misdiagnosis was lower for gadobenate dimeglumine $(2.6 \%, 4.0 \%, 3.5 \%$ for gadobenate dimeglumine vs $4.9 \%, 6.6 \%$, and $11.9 \%$ for gadopentetate dimeglumine; readers 1,2 , and 3 , respectively). Further analysis of data from this study revealed significant superiority for gadobenate dimeglumine not only for lesion detection across different malignant lesion types, but also across different categories of breast parenchyma density. ${ }^{26}$ Moreover, highly significant superiority for malignant lesion detection was noted compared to conventional mammography and ultrasound (Figures 1 to 3 ). ${ }^{26}$

The results of all these intra-individual crossover studies ${ }^{23-26}$ can be ascribed to the greater $\mathrm{r} 1$ relaxivity of gadobenate dimeglumine compared to gadopentetate dimeglumine
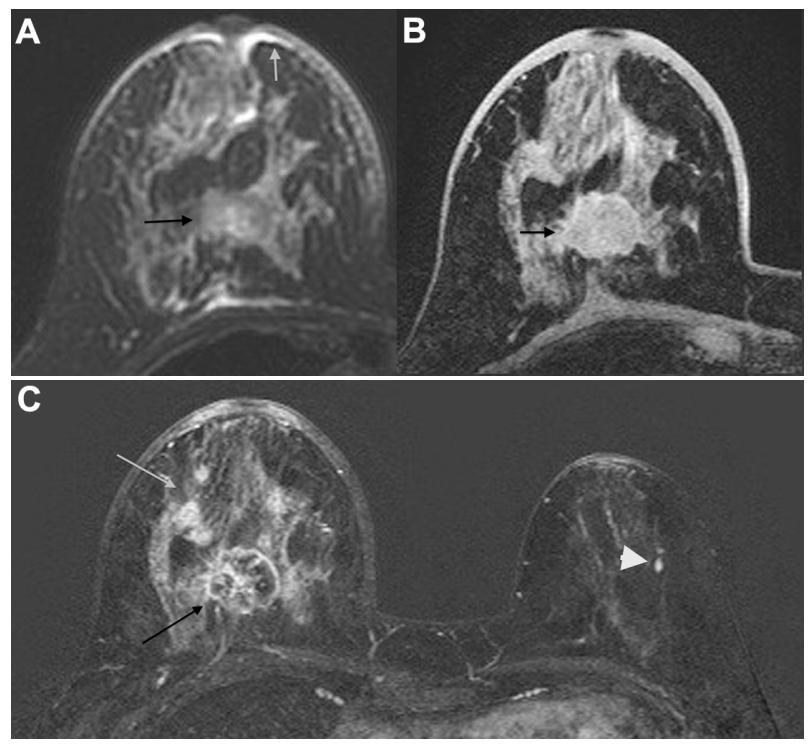

Figure I 48-year-old woman with BI-RADS 6 lesions in the right breast detected on $X R$ mammography and ultrasound (triple negative, IDC).

Notes: Woman underwent breast MRI with gadobenate dimeglumine (MultiHance ${ }^{\circledR}$;) for local staging. MRI detected more nodules in the right breast than conventional imaging and allowed a better evaluation of skin thickness. It was considered multicentric disease and neoadjuvant chemotheraphy was planned. Breast MRI also revealed an enhancing lesion in the left breast, not detected on conventional imaging; second look ultrasound was performed and histological examination proved a DCIS: (A-B) IDEAL water only T2w sequence, axial plane. The grey arrow shows skin thickness while black arrows indicate a round, hyperintense nodule in the right breast. VIBRANT TI w precontrast sequence, axial plane, 3T magnet. (C) VIBRANT TIw dynamic sequence, axial plane, $3 \mathrm{~T}$ magnet. The lesion between inferior quadrants of the right breast has a central area of necrosis (black arrow) and multiple nodules are detectable in the ipsilateral breast (grey arrow). In the left breast there is an enhancing nodule (arrowhead).

Abbreviations: MRI, magnetic resonance imagining; BI-RADS, Breast imagingreporting and data system; IDC, invasive ductal carcinoma; XR, X-ray; DCIS, ductal carcinoma in situ.
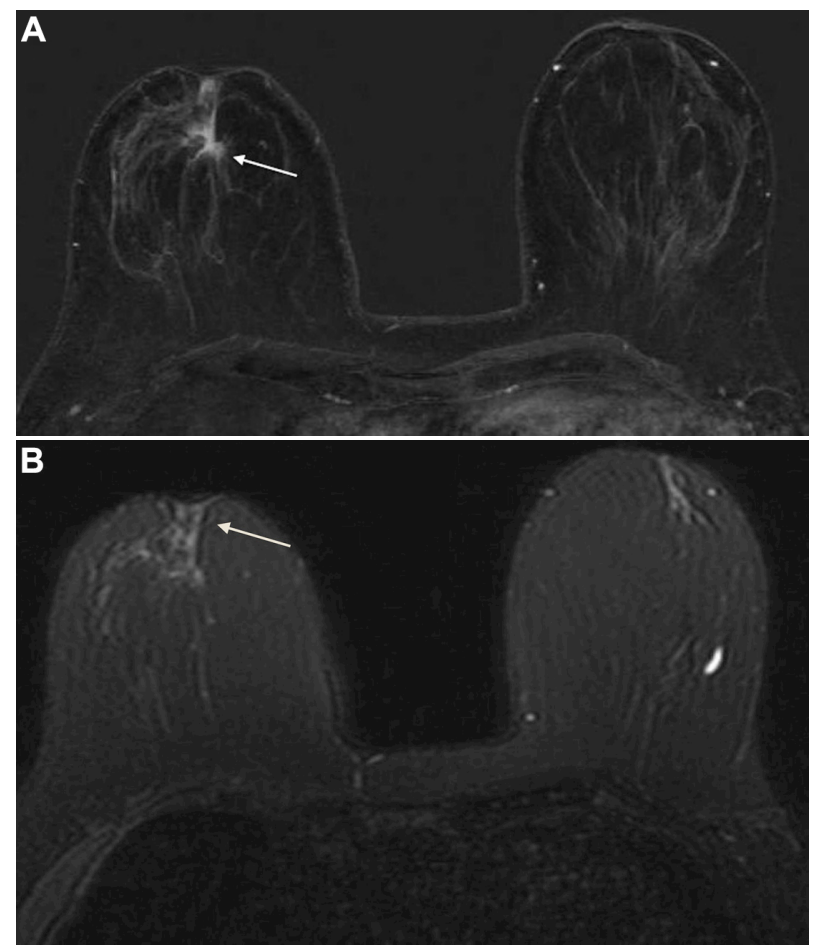

Figure 2 38-year-old woman with nipple retraction.

Notes: Ultrasound did not detect any lesions. Breast MRI with gadobenate dimeglumine (MultiHance) confirmed nipple retraction and detected non masslike enhancement with nipple involvement. (A) TIw sequence, axial plane. Nipple retraction is due to retroareolar non-mass enhancement (white arrow). IS/T curve type II-III. The patient underwent surgery and it was proven to be IDC. (B) T2w water only sequence, nipple retraction is recognizable (white arrow).

Abbreviations: MRI, magnetic resonance imagining; IDC, invasive ductal carcinoma.

(6.2 L/mmols ${ }^{-1}$ vs $4.2 \mathrm{~L} / \mathrm{mmols}^{-1}$ at $\left.1.5 \mathrm{~T}\right) .{ }^{21}$ Since $\mathrm{r} 1$ relaxivity is the single most important parameter defining contrast efficacy in terms of signal intensity enhancement, similar superiority for gadobenate dimeglumine can be anticipated for intra-individual comparisons of this agent with other GBCAs that have $\mathrm{r} 1$ relaxivity values similar to that of gadopentetate dimeglumine.

That breast MRI is demonstrably superior to conventional imaging techniques was further shown by Pediconi et al in 2007 in a study in 118 patients with unilateral breast cancer or high-risk lesions and negative findings in the contralateral breast. ${ }^{31}$ Breast MRI with $0.1 \mathrm{mmol} / \mathrm{kg}$ gadobenate dimeglumine at $1.5 \mathrm{~T}$ resulted in the detection of contralateral lesions in $28(24 \%)$ patients, predominantly in women with dense breast parenchyma. The sensitivity, specificity, accuracy, and positive and negative predictive values of gadobenate dimeglumine-enhanced breast MRI for depiction of malignant or high-risk contralateral lesions were $100 \%, 94 \%, 95 \%, 79 \%$, and $100 \%$, respectively. In a similar study Viehweg et $\mathrm{al}^{32}$ obtained sensitivity, specificity, and accuracy values of $91 \%$, 

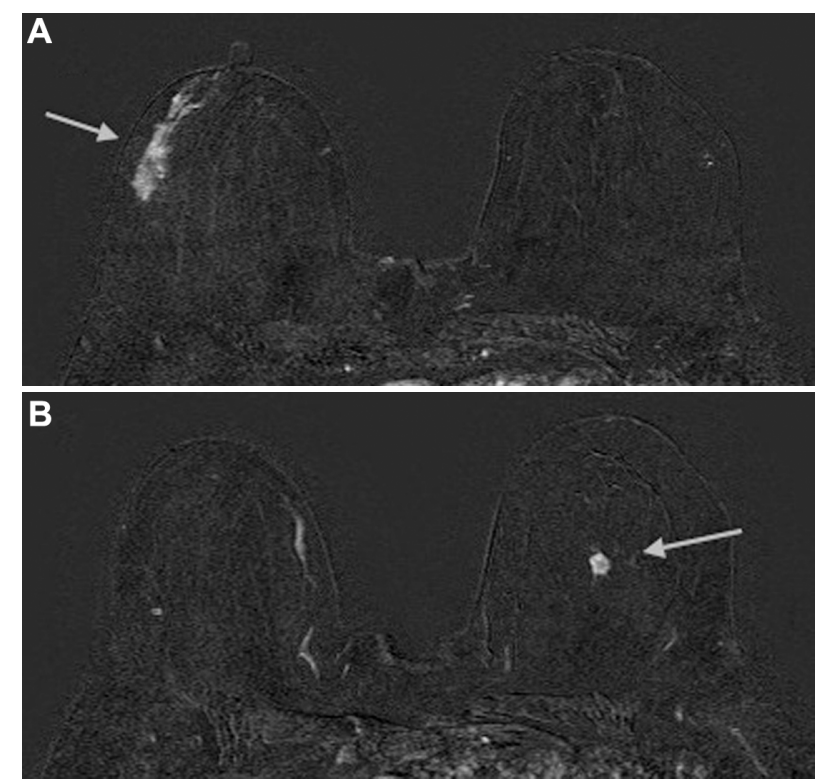

Figure 3 Breast MRI of a 59-year-old woman revealed a greater extension of the lesion on the right breast than $\mathrm{XR}$ mammography and ultrasound. At pathology both lesions were proved to be invasive ductal carcinoma.

Notes: TIw sequence, axial plane. Breast MRI with gadobenate dimeglumine (MultiHance) allowed detection of two lesions in both breasts. Non-mass enhancement in the right breast was previously underestimated at XR mammography and ultrasound. (A) TIw sequence after gadobenate dimeglumine (MultiHance) Non-mass enhancement (grey arrow); type III curve (B) TIw sequence after contrast agent. Mass lesion (grey arrow). Type III curve.

Abbreviations: $M R I$, magnetic resonance imagining; $X R, X$-ray.

$90 \%$, and $90 \%$, respectively, for the detection of contralateral malignant lesions but used a double $(0.2 \mathrm{mmol} / \mathrm{kg})$ dose of gadopentetate dimeglumine.

Although these previous studies have all demonstrated diagnostic superiority for gadobenate dimeglumine, the choice of alternative contrast agents is an important issue in daily practice. For this reason Pediconi et al performed an intra-individual randomized comparison of gadobutrol versus gadobenate dimeglumine..$^{33}$

The study included 72 patients, each of whom underwent two preoperative breast MRI examinations with gadobenate dimeglumine and gadobutrol. No significant differences were observed between the two contrast agents in terms of lesion detection, Breast Imaging-Reporting and Data System assessment or lesion enhancement and morphology and the authors concluded that gadobutrol is non-inferior to gadobenate dimeglumine for breast cancer staging. ${ }^{33}$ However, unlike the comparisons of gadobenate dimeglumine and gadopentetate dimeglumine ${ }^{22-26}$ this study was open to criticism in terms of the patient inclusion criteria and the manner in which the study end-points were selected and analyzed. ${ }^{34}$

\section{Unique features of gadobenate dimeglumine for breast MRI}

In the DETECT tria ${ }^{25}$ no statistically significant differences were observed between gadobenate dimeglumine and gadopentetate dimeglumine in terms of the appearance of the signal intensity-time curves for individual lesions although the magnitude of contrast enhancement was significantly higher with gadobenate dimeglumine. ${ }^{25} \mathrm{~A}$ potential benefit of this higher contrast enhancement is improved diagnostic performance relative to breast MRI with lower relaxivity GBCAs. In looking to investigate the potential for improved diagnostic performance Sardanelli et a ${ }^{35}$ evaluated 34 patients with 36 breast lesions and ascribed a score based on shape (round/oval/lobular $=0$; linear/dendritic/stellate $=1$ ), margins (defined $=0$; undefined $=1$ ), enhancement pattern (homogeneous $=0$; inhomogeneous $=1$; rim $=2$ ), kinetics (continuous $=0$; plateau $=1$; washout $=2$ ), and initial enhancement. For the assessment of initial enhancement a standard threshold score (ie, $<50 \%=0 ; 50 \%-100 \%=1$; $>100 \%=2$ ) was compared with an adjusted threshold score in which $<100 \%=0 ; 100 \%-240 \%=1 ;>240 \%=2$. Overall, 33 lesions (26 malignant and seven benign) had an initial enhancement higher than $100 \%$, of which 17 had initial enhancement greater than $240 \%$. Application of adjusted thresholds led to improved specificity $(75 \%$ with adjusted thresholds vs $13 \%$ with standard thresholds) without affecting sensitivity (96\%) for malignant lesion detection. Values for accuracy, positive predictive value, and negative predictive value were also better with adjusted thresholds (92\%, 93\% and $86 \%$, respectively, with adjusted thresholds vs $78 \%, 79 \%$, and $50 \%$ respectively, with standard thresholds). A subsequent study in 68 patients with 73 lesions revealed similar results: ${ }^{36}$ the initial enhancement for benign lesions was $141 \% \pm 65 \%$ (mean \pm standard deviation) compared with $210 \% \pm 80 \%$ $(P=0.001)$ for malignant lesions resulting in improved specificity, accuracy, positive predictive value, and negative predictive value without affecting the sensitivity $(100 \%)$ for malignant lesion detection.

A second benefit of the higher relaxivity of gadobenate dimeglumine for breast MRI is the possibility for improved malignant lesion detection based on an association between cancer and increased breast vascularity. ${ }^{37-39}$ Gadobenate dimeglumine is ideally suited to vascular imaging because of its greater $r 1$ relaxivity derived from interaction with human serum albumin. Since neoangiogenesis and increased breast vascularity is associated with breast cancer, improved depiction of ipsilateral increased vascularity is highly indicative of 

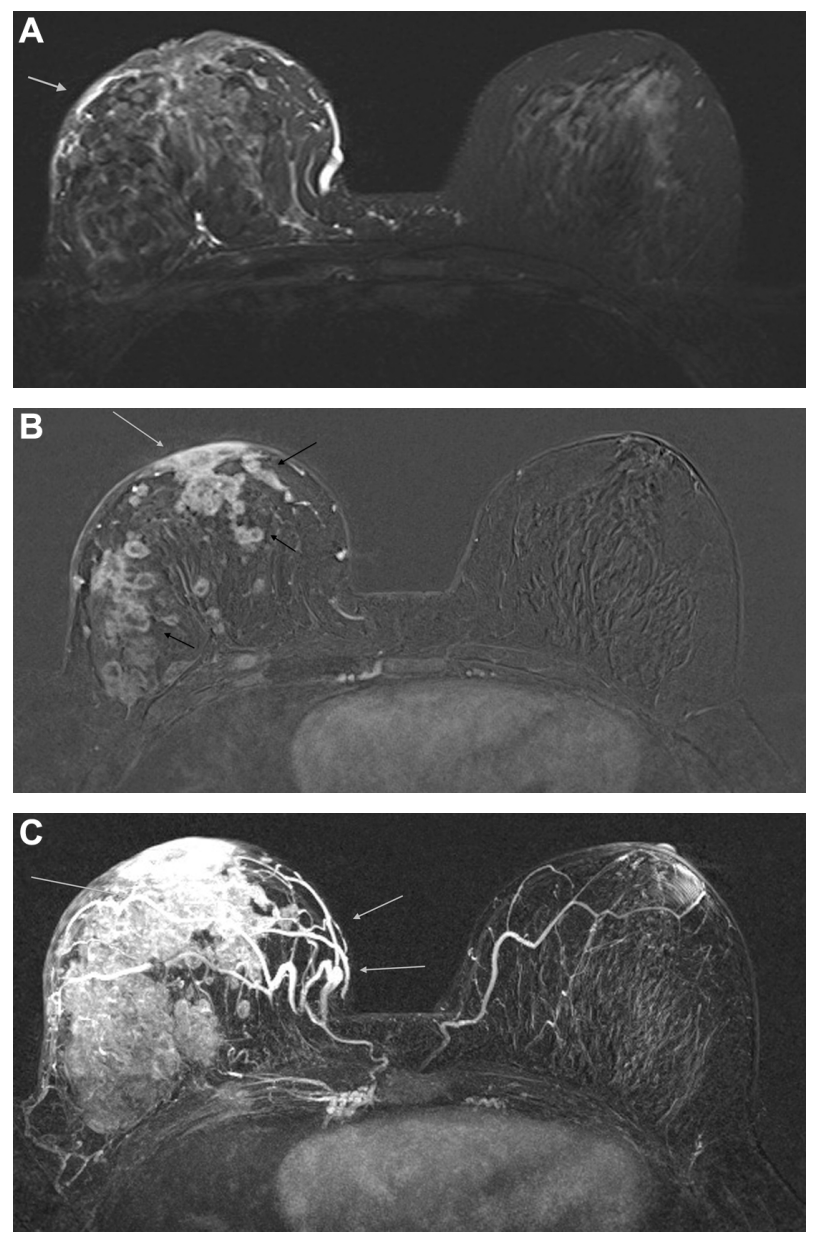

Figure 4 A 53-year-old woman with multiple lesions of the right breast, as seen on a breast ultrasound, proved to be invasive ductal carcinoma at pathology. She underwent a breast MRI to determine the extent of the disease and the multicentricity.

Notes: (A) T2w sequence, axial plane, I.5T magnet. Asymmetry between breasts and skin thickness (grey arrow) of the right breast are recognizable. (B) TIw dynamic sequence. After gadobenate dimeglumine (Multihance) administration it is possible to detect multiple enhancing lesions (black arrows) in the right breast. Moreover skin and nipple-areola complex (grey arrow) involvement are well appreciable. IS/T curve type III. (C) Maximum intensity projection (MIP) images in the axial plane. The right breast shows increased vascularity (grey arrows).

Abbreviations: MRI, magnetic resonance imagining.

malignancy (Figure 4). A study by Sardanelli et al revealed better evaluation of breast vessels and improved detection of one-sided increased vascularity with $0.1 \mathrm{mmol} / \mathrm{kg}$ gadobenate dimeglumine than with $0.1 \mathrm{mmol} / \mathrm{kg}$ gadopentetate dimeglumine. ${ }^{39}$ Specifically, 95 women with known or suspected breast cancer underwent breast MRI, receiving either gadobenate dimeglumine at a dose of $0.05,0.1$ or 0.2 $\mathrm{mmol} / \mathrm{kg}$ bodyweight or gadopentetate dimeglumine at a dose of $0.1 \mathrm{mmol} / \mathrm{kg}$ bodyweight. Assessment was then made of maximum intensity projection images since these images, obtained by post-processing subtracted (post-contrast pre-contrast) breast images, reveal not only the presence of enhanced lesions but also the angiographic vascular map of vessels within the breast. A score ranging from 0 , indicating absent or very low breast vascularity, to 3, indicating high breast vascularity, was assigned to the maximum intensity projection images on the basis of the number of vessels seen and the length and conspicuity of the vessels. Breast increased vascularity was assumed when the difference in the number of vessels between the two breasts was two or more. Overall, 67 patients demonstrated asymmetric breast vascularity due to the presence of one-sided increased vascularity. Statistically significant differences in vascular map scores were observed between the three gadobenate dimeglumine dose groups and the gadopentetate dimeglumine dose group. Notably, significant differences between the gadobenate dimeglumine groups were not observed; a dose of just $0.05 \mathrm{mmol} / \mathrm{kg}$ bodyweight was shown to produce high quality vascular maps of the breast and that the angiographic effect at this and higher doses is significantly greater than the effect following the administration of a standard $0.1 \mathrm{mmol} / \mathrm{kg}$ dose of gadopentetate dimeglumine. This study confirmed the association between vascular map asymmetry and ipsilateral invasive breast cancer with sensitivity and specificity values of $88 \%$ and $82 \%$, respectively, for ipsilateral malignancy. ${ }^{39}$

\section{Possible adverse effects of gadobenate dimeglumine}

Although GBCAs have long been considered extremely safe, their safety has been of growing concern following the advent of nephrogenic systemic fibrosis (NSF). ${ }^{40} \mathrm{NSF}$ is a rare disease which occurs in patients with severe renal impairment, particularly in the presence of a proinflammatory process. Available data do not reveal an increased risk of NSF for gadobenate dimeglumine (no unconfounded cases of NSF have been reported with this agent) and it is considered safer than other GBCAs, such as gadodiamide and gadopentetate dimeglumine, in patients with renal impairment. ${ }^{41-43}$ Numerous studies reveal a robust safety profile for this agent.

\section{Conclusion}

A number of studies have shown that gadobenate dimeglumine is superior to other GBCAs for breast MRI in allowing improved diagnostic performance.

The use of gadobenate dimeglumine for breast MRI is preferable to other GBCAs. Its higher relaxivity helps improve the detection and characterization of malignant breast lesions at an equivalent dose to that of other GBCAs such as gadopentetate dimeglumine. Based on available data the identification of breast lesions is improved with the use of gadobenate dimeglumine and the rate of cancer misdiagnosis is lower. 


\section{Disclosure}

Miles A Kirchin is an employee of Bracco Imaging SpA. The other authors report no conflicts of interest in this work.

\section{References}

1. Saslow D, Boetes C, Burke W, et al. American Cancer Society guidelines for breast screening with MRI as an adjunct to mammography. CA Cancer J Clin. 2007;57(2):75-89.

2. Bary F, McCarron P, Parkin DM. The changing global patterns of female breast cancer incidence and mortality. Breast Cancer Res. 2004;6(6): 229-239.

3. Akinyemiju TF, Genkinger JM, Farhat M, et al. Residential environment and breast cancer incidence and mortality: a systematic review and meta-analysis. BMC Cancer. 2015;15:191.

4. Van den Broek AJ, Schmidt MK, van't Veer LJ, Tollenaar RA, van Leeuwen FE. Worse breast cancer prognosis of BRCA1/BRCA2 mutation carriers: what's the evidence? A systematic review with meta-analysis PLoS One. 2015;10(3):e0120189.

5. Warner E, Messermith H, Causer P, Eisen A, Shumak R, Plewes D. Systematic review: using magnetic resonance imaging to screen women at high risk for breast cancer. Ann Intern Med. 2008;148(9):671-679.

6. Brem RF, Leniham MJ, Lieberman J, Torrente J. Screening breast ultrasound: past, present and future. AJR Am J Roentgenol. 2015;204(2): 234-240.

7. Kuhl CK, Schrading S, Leutner CC, et al. Mammography, breast ultrasound and magnetic resonance imaging for surveillance of women at high familial risk for breast cancer. J Clin Oncol. 2005;23(33):8469-8476.

8. Millet I, Pages E, Hoa D. Pearls and pitfalls in breast MRI. Br J Radiol. 2012;85(1011):197-207.

9. Berg WA, Gutierrez L, NessAiver MS, et al. Diagnostic accuracy of mammography, clinical examination, US, and MR imaging in preoperative assessment of breast cancer. Radiology. 2004;233(3):830-849.

10. Peters NH, Borel Rinkes IH, Zuitoff NP, et al. Meta-analysis of MR imaging in the diagnosis of breast lesions. Radiology. 2008;246(1): $116-124$.

11. Le-Petross HT, Shetty MK. Magnetic resonance imaging and breast ultrasonography as an adjunct to mammographic screening in high-risk patients. Semin Ultrasound CT MR. 2011;32(4):266-272.

12. Tharmaratnam K, Hagen AI, Moller P. MRI screening of women with hereditary predisposition to breast cancer: diagnostic performance and survival analysis. Breast Cancer Res Treat. 2014;148(3):687-688.

13. Oztekin PS, Kosar PN. Magnetic Resonance Imaging of the breast as a problem solving method: to be or not to be? Breast J. 2014;20(6):622-631.

14. Sung JS, Li J, Da Costa G, et al. Preoperative breast MRI for early stage breast cancer: effect on surgical and long term outcomes. AJR Am J Roentgenol. 2014;2012(6):1376-1382.

15. Houssami N, Turner R, Morrow M. Preoperative magnetic resonance imaging in breast cancer: meta-analysis of surgical outcomes. Ann Surg. 2013;257(2):249-255.

16. Gonzalez V, Sandelin K, Karlsson A, et al. Preoperative MRI of the breast (POMB) influences primary treatment in breast cancer: a prospective, randomized, multicenter study. World J Surg. 2014;38(7):1685-1693.

17. Sardanelli F, Podo F, Santoro F, et al. Multicenter surveillance of women at high genetic breast cancer risk using mammography, ultrasonography, and contrast-enhanced magnetic resonance imaging (the high breast cancer risk italian 1 study): final results. Invest Radiol. 2011;46(2):94-105.

18. Mann RM, Balleyguier C, Baltzer PA, et al. Breast MRI: EUSOBI recommendations for women's information. Eur Radiol. Epub 2015 May 23.

19. Bellin MF, Van Der Molen AJ. Extracellular gadolinium-based contrast media: an overview. Eur J Radiol. 2008;66(2):160-167.

20. Bellin MF. MR contrast agents, the old and the new. Eur J Radiol. 2006;60(3):314-323.

21. Shen Y, Goerner FL, Snyder C, et al. T1 Relaxivities of Gadolinium Based Magnetic Resonance contrast agents in human whole blood at 1.5, 3 and 7 T. Invest Radiol. 2015;50(5):330-338.
22. Knopp MV, Bourne MW, Sardanelli F, et al. Gadobenate dimeglumineenhanced MRI of the breast: analysis of dose response and comparison with gadopentetate dimeglumine. AJR Am J Roentgenol. 2003;181(3): 663-676.

23. Pediconi F, Catalano C, Occhiato R, et al. Breast lesion detection and characterization at contrast enhanced MR mammography: gadobenate dimeglumine versus gadopentetate dimeglumine. Radiology. 2005; 237(1):45-56.

24. Pediconi F, Catalano C, Padula S, et al. Contrast-enhanced MR mammography: improved lesion detection and differentiation with gadobenate dimeglumine. AJR Am J Roentgenol. 2008;191(5): 1339-1346.

25. Martincich L, Faivre-Pierret M, Zechmann MC, et al. Multicenter, double-blind, randominzed, intraindividual crossover comparison of gadobenate dimeglumine and gadopentetate dimeglumine for breast MR imaging (DETECT Trial). Radiology. 2011;258(2):396-408.

26. Gilbert FJ, van den Bosch HC, Petrillo A, et al. Comparison of gadobenate dimeglumine-enhanced breast MRI and gadopentetate dimeglumine-enhanced breast MRI with mammography and ultrasound for the detection of breast cancer. J Magn Reson Imaging. 2014;39(5):1272-1286.

27. Heywang SH, Hahn D, Schimdt H, et al. MR imaging of the breast using gadolinium DTPA. J Comput Assist Tomogr. 1986;10(2):199-204.

28. Renz DM, Durmus T, Bottcher J, et al. Comparison of gadoteric acid and gadobutrol for detection as well as morphologic and dynamic characterization of lesions on breast dynamic contrast enhanced magnetic resonance imaging. Invest Radiol. 2014;49(7):474-484.

29. Fallenberg EM, Renz DM, Karle B, et al. Intraindividual randomized comparison of the macrocyclic contrast agent gadobutrol and gadoterate meglumine in breast magnetic resonance. Eur Radiol. 2015;25(3): 837-849.

30. Carbonaro LA, Pediconi F, Verardi N, et al. Breast MRI using a high relaxivity contrast agent: an overview. AJR Am J Roentgenol. 2011 196(4):942-955.

31. Pediconi F, Catalano C, Roselli A, et al. Contrast enhanced MR mammography for the evaluation of contralateral breasts in patients with diagnosed unilateral breast cancer or high-risks lesions. Radiology. 2007;243(3):670-680.

32. Viehweg P, Rotter K, Laniado M, et al. MR imaging of the contralateral breast in patients after breast-conserving therapy. Eur Radiol. 2004;14(3):402-408.

33. Pediconi F, Kubik-Huch R, Chilla B, Schwenke C, Kinkel K Intra-individual randomized comparison of gadobutrol $1.0 \mathrm{M}$ versus gadobenate dimeglumine $0.5 \mathrm{M}$, in patients scheduled for preoperative breast MRI. Eur Radiol. 2013;23(1):84-92.

34. Schneider G, Fries P. Intra-individual randomised comparison of gadobutrol 1.0 M versus gadobenate dimeglumine $0.5 \mathrm{M}$ in patients scheduled for preoperative breast MRI. Eur Radiol. 2013;23(8): 2095-2096.

35. Sardanelli F, Fausto A, Esseridou A, Di Leo G, Kirchin MA. Gadobenate dimeglumine as a contrast agent for dynamic breast magnetic resonance imaging: effect of higher initial enhancement thresholds on diagnostic performance. Invest Radiol. 2008;43(4):236-242.

36. Carbonaro LA, Verardi N, Di Leo G, Sardanelli F. Handling a high relaxivity contrast material for dynamic breast MR imaging using higher thresholds for the initial enhancement. Invest Radiol. 2010;45(3): 114-120.

37. Mahfouz AE, Sherif H, Saad A, et al. Gadolinium-enhanced MR angiography of the breast: is breast cancer associated with ipsilateral higher vascularity? Eur Radiol. 2001;11(6):965-969.

38. Carriero A, Di Credico A, Mansour M, Bonomo L. Maximun intensity projection analysis in magnetic resonance of the breast. $J$ Exp Clin Cancer Res. 2002;21(3 Suppl):77-81.

39. Sardanelli F, Iozzelli A, Fausto A, Carriero A, Kirchin MA. Gadobenate dimeglumine-enhanced MR imaging breast vascular maps: association between invasive cancer and ipsilateral higher vascularity. Radiology. 2005;235(3):791-797. 
40. Van der Molen AJ. Nephrogenic systemic fibrosis and the role of gadolinium contrast media. $J$ Med Imaging Radiat Oncol. 2008;52(4):339-350.

41. Heverhagen JT, Krombach GA, Gizewski E. Application of extracellular gadolinium-based MRI contrast agents and the risk of nephrogenic systemic fibrosis. Rofo. July 2014;186(7):661-669.

42. Soulez G, Bloomgarden DC, Rofsky NM, et al. Prospective Cohort Study of Nephrogenic Systemic Fibrosis in Patients With Stage 3-5 Chronic Kidney Disease Undergoing MRI With Injected Gadobenate Dimeglumine or Gadoteridol. Am J Roentgenol. 2015;205(3):469-478.
43. Nandwana SB, Moreno CC, Osipow MT, Sekhar A, Cox KL. Gadobenate dimeglumine administration and nephrogenic systemic fibrosis: is there a real risk in patients with impaired renal function? Radiology. 2015;276(3):741-747.

\section{Publish your work in this journal}

Research and Reports in Nuclear Medicine is an international, peerreviewed, open access journal publishing original research, reports, reviews and commentaries on all areas of nuclear medicine. The manuscript management system is completely online and includes a very quick and fair peer-review system. Visit http://www.dovepress.com/ testimonials.php to read real quotes from published authors. 